TITLE:

\title{
Ultrafast free-carrier dynamics in Cu2ZnSnS4 single crystals studied using femtosecond time-resolved optical spectroscopy
}

\section{$\operatorname{AUTHOR}(\mathrm{S})$ :}

Phuong, L. Q.; Okano, M.; Yamada, Y.; Yamashita, G.; Morimoto, T.; Nagai, M.; Ashida, M.; Nagaoka, A.; Yoshino, K.; Kanemitsu, Y.

\section{CITATION:}

Phuong, L. Q.... [et al]. Ultrafast free-carrier dynamics in Cu2ZnSnS4 single crystals studied using femtosecond time-resolved optical spectroscopy. Applied Physics Letters 2014, 105(23): 231902.

ISSUE DATE:

2014-12-08

URL:

http://hdl.handle.net/2433/192276

\section{RIGHT:}

Copyright 2014 American Institute of Physics. This article may be downloaded for personal use only. Any other use requires prior permission of the author and the American Institute of Physics. 


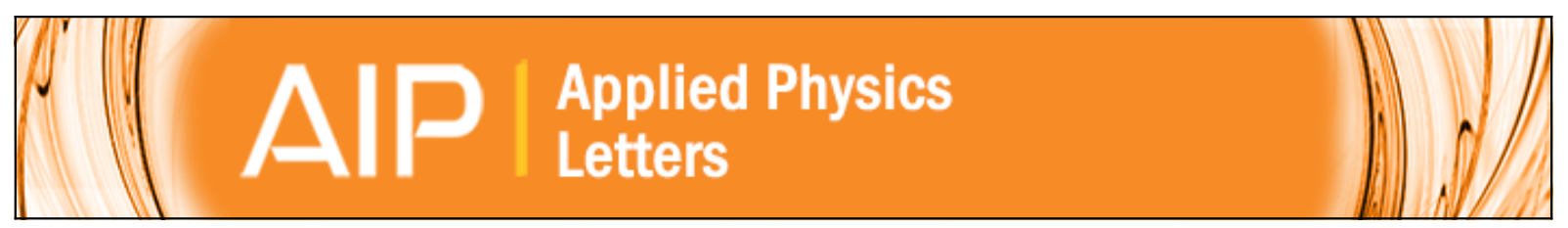

\section{Ultrafast free-carrier dynamics in Cu2ZnSnS4 single crystals studied using femtosecond time-resolved optical spectroscopy}

L. Q. Phuong, M. Okano, Y. Yamada, G. Yamashita, T. Morimoto, M. Nagai, M. Ashida, A. Nagaoka, K. Yoshino , and Y. Kanemitsu

Citation: Applied Physics Letters 105, 231902 (2014); doi: 10.1063/1.4903802

View online: http://dx.doi.org/10.1063/1.4903802

View Table of Contents: http://scitation.aip.org/content/aip/journal/apl/105/23?ver=pdfcov

Published by the AIP Publishing

\section{Articles you may be interested in}

Terahertz surface emission from Cu2ZnSnSe4 thin film photovoltaic material excited by femtosecond laser pulses

Appl. Phys. Lett. 105, 231104 (2014); 10.1063/1.4903740

Employing time-resolved terahertz spectroscopy to analyze carrier dynamics in thin-film Cu2ZnSn(S,Se)4 absorber layers

Appl. Phys. Lett. 104, 253901 (2014); 10.1063/1.4884817

Temperature-dependent photocarrier recombination dynamics in Cu2ZnSnS4 single crystals

Appl. Phys. Lett. 104, 081907 (2014); 10.1063/1.4866666

Photocarrier localization and recombination dynamics in Cu2ZnSnS4 single crystals

Appl. Phys. Lett. 103, 191902 (2013); 10.1063/1.4829063

Size and surface effects on transient photoconductivity in CdS nanobelts probed by time-resolved terahertz spectroscopy

Appl. Phys. Lett. 101, 091104 (2012); 10.1063/1.4748300

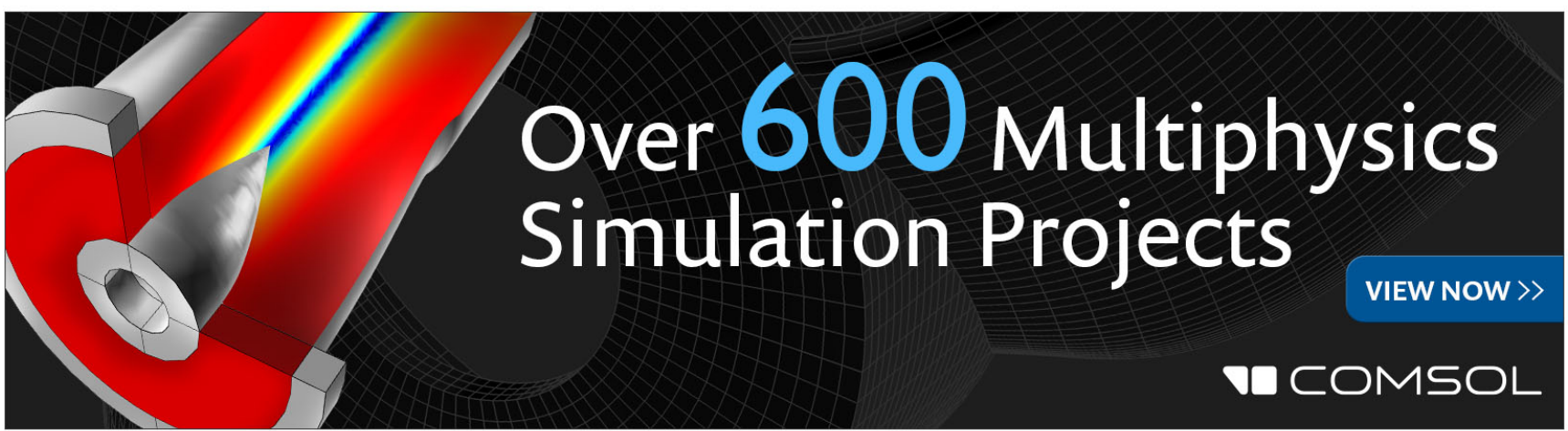




\title{
Ultrafast free-carrier dynamics in $\mathrm{Cu}_{2} \mathrm{ZnSnS}_{4}$ single crystals studied using femtosecond time-resolved optical spectroscopy
}

\author{
L. Q. Phuong, ${ }^{1,2}$ M. Okano, ${ }^{1}$ Y. Yamada, ${ }^{1}$ G. Yamashita, ${ }^{3}$ T. Morimoto, ${ }^{3}$ M. Nagai, ${ }^{3}$ \\ M. Ashida, ${ }^{3}$ A. Nagaoka, ${ }^{4,5}$ K. Yoshino, ${ }^{5}$ and Y. Kanemitsu ${ }^{1,2, a)}$ \\ ${ }^{1}$ Institute for Chemical Research, Kyoto University, Uji, Kyoto 611-0011, Japan \\ ${ }^{2}$ Japan Science and Technology Agency, CREST, Uji, Kyoto 611-0011, Japan \\ ${ }^{3}$ Graduate School of Engineering Science, Osaka University, Osaka 560-8531, Japan \\ ${ }^{4}$ Department of Materials Science and Engineering, Kyoto University, Kyoto 606-8501, Japan \\ ${ }^{5}$ Department of Applied Physics and Electronic Engineering, University of Miyazaki, \\ Miyazaki 889-2192, Japan
}

(Received 6 October 2014; accepted 24 November 2014; published online 9 December 2014)

\begin{abstract}
We studied the dynamics of photogenerated carriers in $\mathrm{Cu}_{2} \mathrm{ZnSnS}_{4}$ (CZTS) single crystals using femtosecond transient reflectivity (TR) and optical pump-THz probe transient absorption (THzTA) spectroscopy. The TR and THz-TA decay dynamics consistently showed that free carriers have long lifetimes of up to a few nanoseconds. The excitation-photon-energy-dependent TR measurements revealed a slow picosecond energy relaxation of free carriers to the band edge in CZTS. The relaxation and recombination dynamics of free carriers were affected by nonradiative recombinations at the surface. Our results revealed a global feature of energy relaxation and recombination processes of free carriers in CZTS single crystals. (C) 2014 AIP Publishing LLC.
\end{abstract}

[http://dx.doi.org/10.1063/1.4903802]

$\mathrm{Cu}_{2} \mathrm{ZnSnS}_{4}$ (CZTS), which is composed of earthabundant and low-toxicity elements, is being considered as a photovoltaic material for next-generation low-cost solar cells $^{1,2}$ owing to its optimal band gap for solar-energy conversion and high absorption coefficient in the near-infrared and visible spectral regions. ${ }^{3,4}$ However, the maximum power conversion efficiency of CZTS-based solar cells is only $\sim 8.4 \% ;^{5}$ it must be improved significantly to realize practical applications. A more insightful understanding of the fundamental optoelectronic properties that govern the photovoltaic operation of solar cells is a prerequisite for improving the power conversion efficiency, and most of the previous studies have been devoted to the physical phenomena related to complicated defects in CZTS. ${ }^{6-10}$ Theoretical and experimental studies have revealed that the defects formed in CZTS are located rather deeper in the forbidden gap than those in $\mathrm{CuIn}_{1-\mathrm{x}} \mathrm{Ga}_{\mathrm{x}} \mathrm{Se}$ (CIGS), ${ }^{11}$ resulting in a low open-circuit voltage in CZTS-based solar cells. ${ }^{5}$

Thus far, the knowledge of free-carrier dynamics in CZTS, which plays a critical role in photovoltaic operation of solar cells, has been scarce. The polycrystalline samples widely used in previous works contain different grains with different sizes, shapes, chemical compositions, etc., and the grain boundary defects usually serve as carrier traps. Therefore, the dynamic behaviors of free carriers in polycrystalline samples are complicated, making it difficult to explore the free-carrier dynamics. Single-crystal samples are favorable for extracting the intrinsic characteristics of freecarrier dynamics, which will be greatly helpful for designing highly efficient thin-film solar cells.

Time-resolved optical spectroscopy provides essential information on photocarrier relaxation and recombination

\footnotetext{
${ }^{\text {a) }}$ Author to whom correspondence should be addressed. Electronic mail: kanemitu@scl.kyoto-u.ac.jp
}

processes in emerging solar cell materials. ${ }^{12,13}$ Particularly, optical pump-optical probe transient reflectivity (TR) and absorption spectroscopic techniques are widely used to investigate the ultrafast sub-picosecond dynamic evolution of photoexcited carriers in various types of bulk materials. ${ }^{14}$ By probing with a $\mathrm{THz}$ pulse, transient dynamics is determined mainly by the temporal change in free-carrier density. ${ }^{15}$ A combination of these two spectroscopic techniques will be fruitful for achieving more precise and detailed understandings of free-carrier dynamics in solar-cell materials.

In this Letter, the dynamics of free carriers in CZTS single crystals has been studied using femtosecond TR and optical pump-THz probe transient absorption (THz-TA) spectroscopy. In both TR and THz-TA experiments, we observed that free carriers have long lifetimes owing to the thermal excitation of localized photocarriers from the tail states below the band edge. In addition, we found a slow energy relaxation of free carriers to the band edge in CZTS. Under high-energy photoexcitation, the dynamics of photogenerated carriers in CZTS is influenced by nonradiative recombinations at the sample surface.

The CZTS single crystals used in this study were grown using the traveling heater method. ${ }^{16}$ The composition of the CZTS single crystals was estimated from the stoichiometric ratio as slightly $\mathrm{Cu}$-poor, $\mathrm{Zn}$-rich, Sn-poor, and S-rich. ${ }^{17}$ The $\mathrm{Cu}$-poor, Zn-rich, and S-rich composition is important to obtain highly efficient CZTS-based solar cells. ${ }^{4}$ These samples were kept at room temperature (RT) when performing all experiments.

In photoluminescence excitation (PLE) and photocurrent (PC) measurements, a wavelength-tunable continuous-wave Ti:sapphire laser was utilized for photoexcitation energies ranging from 1.36 to $1.72 \mathrm{eV}$, while a broadband white-light picosecond laser equipped with a bandpass transmission 
filter was used for photoexcitation energies ranging from 1.51 to $2.21 \mathrm{eV}$. The PL signal was detected using a liquidnitrogen-cooled InGaAs photodiode array through a $30-\mathrm{cm}$ monochromator. The PC collected from two Au electrodes deposited on the sample surface was converted to voltage and then recorded through a lock-in amplifier.

THz-TA measurements were performed using a $1-\mathrm{kHz}$ Ti:sapphire regenerative amplified laser with a pulse duration of $35 \mathrm{fs}^{18}$ The excitation pump with tunable photon energy emitted from an optical parametric amplifier was chopped with a frequency of $250 \mathrm{~Hz}$ and focused loosely on the sample attached to a metal plate with a 2 -mm-diameter hole. A THz probe pulse with a center frequency of $1 \mathrm{THz}$ generated from two-color pumped air plasma was directed to the excitation spot. The electric-field profile of the transmitted THz pulse was detected using the electro-optic sampling method by utilizing a 1-mm-thick ZnTe crystal. The detectable range was from 0.5 to $2.5 \mathrm{THz}$.

In TR measurements, the excitation pulse emitted from an optical parametric amplifier pumped by a Yb:KGW (potassium gadolinium tungstate) regenerative amplified laser with a pulse duration of $\sim 200 \mathrm{fs}$ and a repetition rate of $50 \mathrm{kHz}$ was chopped with a frequency of $130 \mathrm{~Hz}$ and irradiated on the sample. The excitation photon energy was tuned from 1.62 to $3.10 \mathrm{eV}$. A part of the seed pulse from the $\mathrm{Yb}: \mathrm{KGW}$ regenerative amplified laser was focused on a sapphire crystal to generate a white-light probe pulse. The reflection of probe pulses from the excitation spot was collected through a monochromator. The TR signals were obtained by comparing the reflectivity of probe pulses with and without the chopped pump-pulse excitation. While the temporal resolution in THz-TA experiments was a few picoseconds, the TR measurements were able to reveal ultrafast sub-picosecond dynamic responses of photoexcited carriers.

The time evolution of the free carriers generated after photoexcitation can be tracked using either TR or THz-TA spectroscopy. Figure 1(a) shows the THz-TA and TR decay dynamics (where $E_{\text {probe }} \approx E_{g}$ ) under a weak $2.06-\mathrm{eV}$ photoexcitation at RT. The THz-TA decay curves are nonexponential and almost identical to the TR decay curves. This means that both THz-TA and TR decay curves demonstrate time-dependent free-carrier population after photoexcitation. The free carriers in CZTS have long lifetimes of up to a few nanoseconds. Note that a slow nanosecond-scale decay was also observed in the PL kinetic trace that reflects the dynamics of the localized photocarriers. ${ }^{4,9,10}$ Thus, we attribute the long lifetime of free carriers in CZTS to the thermal excitation from the tail states. ${ }^{11,19,20}$

The amplitude of the THz-TA signal just after photoexcitation, which reflects the total number of photoexcited free carriers, is plotted as a function of the photoexcitation energy in Fig. 1(b), along with the PC spectrum obtained using the conventional measurement in which the electrodes on the sample surfaces were utilized to collect the photoexcited carriers. A peak around $1.60 \mathrm{eV}$ appears in the PC spectrum, indicating the near-band-edge transitions. ${ }^{4}$ For high-energy photoexcitation, the $\mathrm{PC}$ decreases quite faster than the THzTA amplitude with increasing excitation energy. These decreases in the PC and THz-TA amplitude are due to the nonradiative recombinations at the surface. ${ }^{4,21}$ Under high-
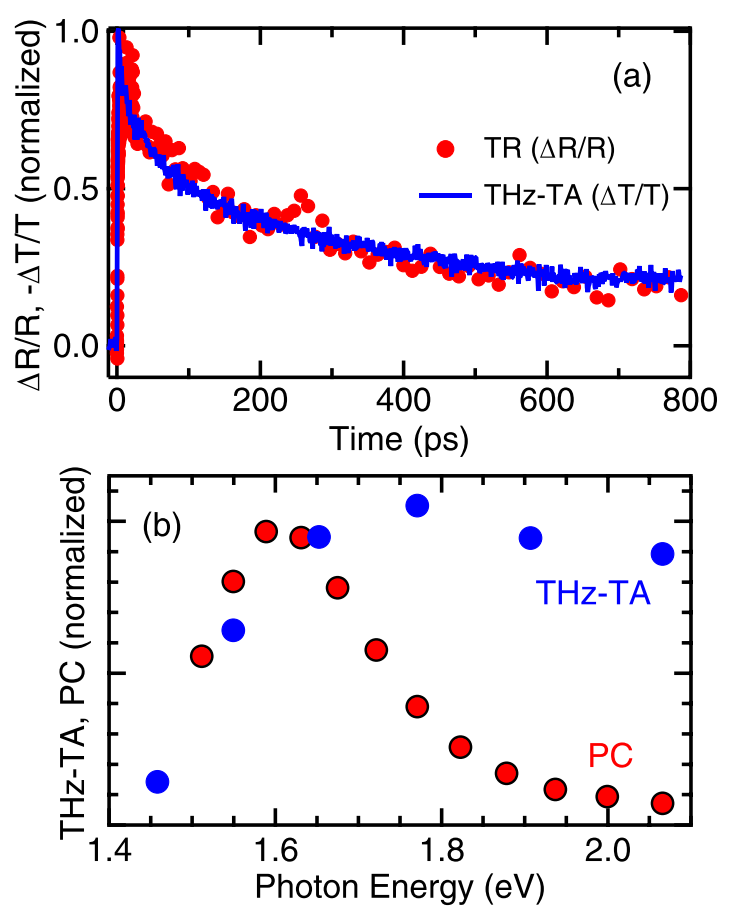

FIG. 1. (a) Transient reflectivity (solid red circles) at a probe energy of $E_{\text {probe }}=1.60 \mathrm{eV}$ and $\mathrm{THz}$ transient absorption (blue curve) decay dynamics under weak 2.06-eV photoexcitation at RT. (b) The amplitude of THz transient absorption just after photoexcitation (THz-TA) as a function of the excitation photon energy (solid blue circles), along with the steady-state PC spectrum (solid red circles).

energy photoexcitation, the generated carriers are located mainly near the sample surface, where the number of nonradiative sites is much larger than that in the bulk. A large ratio of photocarriers is then captured in the nonradiative sites before being collected by the surface electrodes, causing the steeper decrease in the PC compared to the decrease in THzTA amplitude with increasing photoexcitation energy. In order to improve further the charge collection efficiency of CZTS-based solar cells, the nonradiative sites, especially those on the sample surface, must be suppressed.

We now focus on the TR decay dynamics in the early delay time region after photoexcitation to investigate the ultrafast relaxation dynamics of free carriers in CZTS single crystals. In order to proceed with further time-resolved investigations of relaxation dynamics, we first clarify the electronic band structure of CZTS. Figure 2(a) shows a typical PLE spectrum of CZTS single crystal at RT, which exhibits two peaks located at about 1.60 and $1.67 \mathrm{eV}$. The low-energy PLE peak is in good agreement with the observed PC peak, indicating strong absorption due to the near-band-edge transitions. ${ }^{4}$ A schematic of the electronic band structure of CZTS is presented in the inset. The energy separations between the topmost valence band (VB) and the second- and third-topmost VBs of CZTS were theoretically estimated to be about $50-70 \mathrm{meV} .^{22}$ The experimentally observed energy difference between two PLE peaks is consistent with the calculated values. The high-energy PLE peak, therefore, is not due to the high-energy-side drop caused by nonradiative recombination at the surface $e^{4,21}$ but corresponds to the intrinsic features of the CZTS electronic band structure: optical transitions between the lowest conduction band (CB) and the second- and third-topmost VBs. 

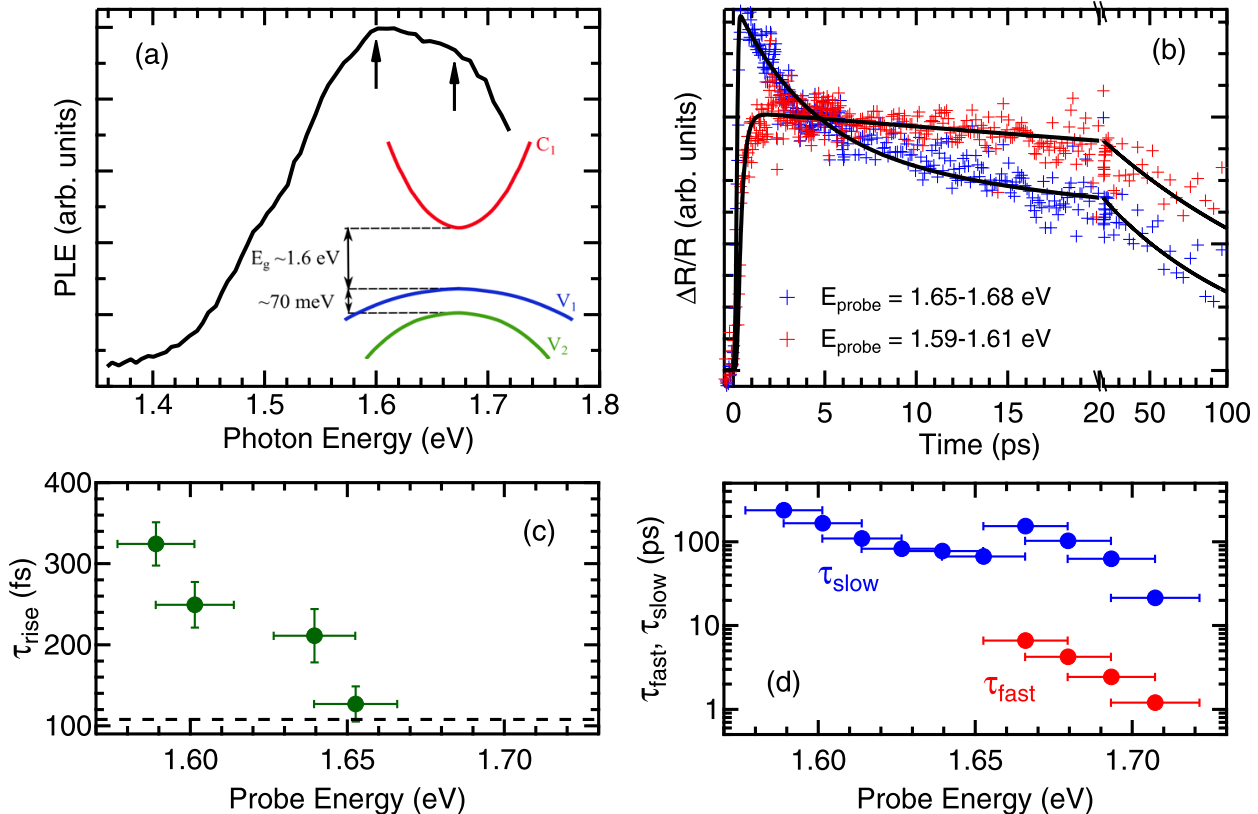

FIG. 2. (a) PLE spectrum of CZTS single crystals at RT. The inset is a schematic of the electronic band structure of CZTS. (b) Transient reflectivity decay dynamics at probe energies of $E_{\text {probe }}=1.59-1.61 \mathrm{eV}$ and $E_{\text {probe }}=1.65-1.68 \mathrm{eV}$ obtained under a weak $1.62-\mathrm{eV}$ excitation at RT. The solid curves are fitting results. (c) Probeenergy dependences of the rise time $\tau_{\text {rise }}$. The dashed line shows the rise time limited by the measurement system. (d) The fast decay time $\tau_{\text {fast }}$ (solid red circles) and slow decay time $\tau_{\text {slow }}$ (solid blue circles) as functions of the probe energy.
Figure 2(b) shows the TR kinetic traces at probe energies of $E_{\text {probe }}=1.59-1.61 \mathrm{eV}$ and $E_{\text {probe }}=1.65-1.68 \mathrm{eV}$ obtained under a weak 1.62-eV photoexcitation at RT, along with their convoluted fitting curves. The TR decay dynamics at low probe energies $\left(E_{\text {probe }}<1.66 \mathrm{eV}\right)$ are composed of one rise and one slow decay components with time constants $\tau_{\text {rise }}$ of several hundreds of femtoseconds and $\tau_{\text {slow }}$ of several tens to hundreds of picoseconds, respectively. Differently, the TR kinetic traces at high probe energies $\left(E_{\text {probe }}>1.66 \mathrm{eV}\right)$ consist of two decay components, fast and slow, with time constants $\tau_{\text {fast }}$ of a few picoseconds and $\tau_{\text {slow }}$ of several tens to hundreds of picoseconds, respectively. The probe energy dependences of $\tau_{\text {rise }}, \tau_{\text {fast }}$, and $\tau_{\text {slow }}$ are summarized in Figs. 2(c) and 2(d).

The nearly resonant $1.62-\mathrm{eV}$ photoexcitation generates electrons only in the lowest CB and holes only in the topmost VB; no photogenerated holes exist in the second- and thirdtopmost VBs. The TR decay dynamics at high probe energies, therefore, reflect the dynamic responses of only the photoexcited electrons in the lowest $\mathrm{CB}$, while the TR decay dynamics at low probe energies should also reflect the dynamic behavior of the holes in the topmost VB. Thus, we attribute the rise time $\tau_{\text {rise }}$, which is observed only in the TR decay dynamics at low probe energies and decreases with increasing probe energy, to the energy relaxation time of photoexcited holes in the topmost VB. The fast decay $\tau_{\text {fast }}$ emerging only in the TR kinetic traces at high probe energies is due to the trapping time of photogenerated electrons to the trap states. The slow decay $\tau_{\text {slow }}$ originates from the nonradiative processes of photocarriers.

In general, the intraband relaxation of photocarriers is governed by carrier-phonon and carrier-carrier scattering. To identify which process is dominant in the energy relaxation of photocarriers to the band edge in CZTS single crystals, we conducted excitation-fluence-dependent TR measurements. Figure 3(a) shows the TR decay dynamics at a probe energy of $E_{\text {probe }}=1.60 \mathrm{eV}\left(\approx E_{g}\right)$ under different fluences of $1.87-\mathrm{eV}$ photoexcitation; the dashed curves are the fitting results. The rise time $\tau_{\text {rise }}$ remains nearly constant as the photoexcitation fluence increases by about one order in magnitude, implying that the energy relaxation of photocarriers in CZTS is predominantly determined by carrier-phonon scattering, rather than carrier-carrier scattering. The electrostatic potential fluctuations occurring in CZTS $^{4,7,9,10}$ result in the spatial separations of photocarriers, which suppresses carrier-carrier scattering. ${ }^{19}$

In order to gain deeper insights into the energy relaxation dynamics of free carriers to the band edge in CZTS, we performed excitation-photon-energy-dependent TR measurements. Figure 3(b) shows the excitation-energy dependence of the TR decay dynamics at a probe energy of $E_{\text {probe }}=1.60 \mathrm{eV}$ obtained under weak photoexcitation. A quickly decaying negative signal appears in TR kinetic traces just after photoexcitation, and a rise time clearly emerges as the excitation photon energy increases. The TR decay dynamics in the early delay time region can then be fitted well using one rise, one decay, and one negative decay components, as shown by the dashed black curves in Fig. 3(b).

Note that the quickly decaying negative TR signal appears clearly only in the photobleaching TR kinetic traces at probe energies around the band-gap energy $E_{g}$ $(\sim 1.60 \mathrm{eV})$. The negative TR signal is thus attributed to the ultrafast band-gap renormalization occurring just after photoexcitation. ${ }^{14}$ In Fig. $3(\mathrm{c})$, the rise time $\tau_{\text {rise }}$ extracted from the fitting results is plotted as a function of the excess energy $E_{\text {exc }}$, which is determined as the energy difference between the pump and probe energies, $E_{\text {pump }}-E_{\text {probe }}$. The energy relaxation of hot carriers to the band edge is slow, up to a few picoseconds, and increases monotonically on increasing the excess energy $E_{\text {exc }}$ (up to $\sim 1 \mathrm{eV}$ ).

The observed energy relaxation rate of hot carriers in CZTS is much slower than those in conventional direct-gap semiconductors, which are typically on the order of tens to hundreds of femtoseconds. ${ }^{23,24}$ Because of spatial distribution of charged defects, electrostatic potential fluctuations occur near the band edge in CZTS ${ }^{4,7,9,10}$ to form local potential minima in the $\mathrm{CB}$ and $\mathrm{VBs} .{ }^{25}$ We believe that these local potential minima and electrostatic effects repeatedly capture the photoexcited carriers during their relaxations to the band edges, therefore, decelerate the energy relaxations of hot 

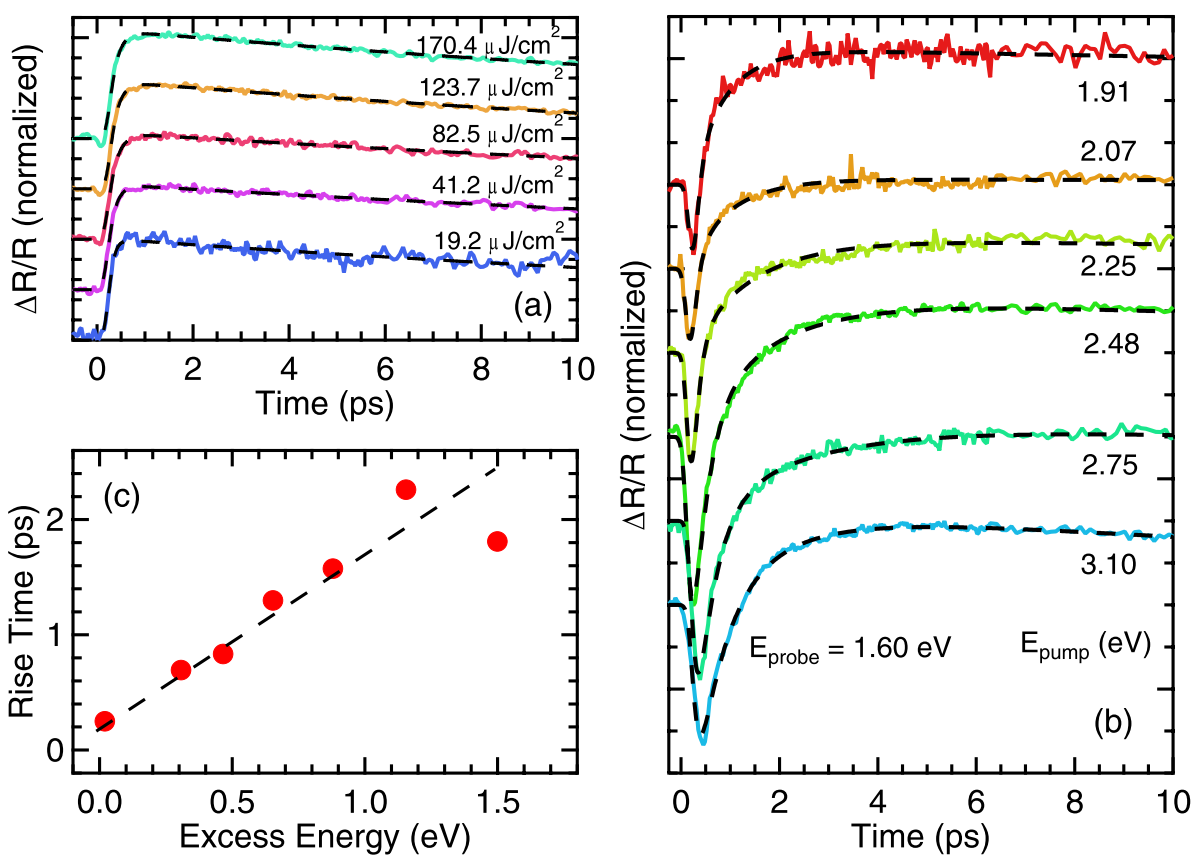

FIG. 3. (a) Transient reflectivity decay dynamics (shifted vertically for clarity) at a probe energy of $E_{\text {probe }}=1.60 \mathrm{eV}$ under different excitation fluences of 1.87-eV photoexcitation. The dashed curves are fitting results. (b) Transient reflectivity decay dynamics (shifted vertically for clarity) at a probe energy of $E_{\text {probe }}=1.60 \mathrm{eV}$ obtained under weak excitations of different photon energies noted nearby. The dashed curves are fitting results. (c) Rise time $\tau_{r i s e}$ detected at a probe energy of $E_{\text {probe }}=1.60 \mathrm{eV}$ as a function of the excess energy. The dashed line is a guide for the eye. carriers. For polycrystalline CIGS in which such potential fluctuations take place, ${ }^{7,26}$ a similar slow relaxation of photocarriers has been recently observed. ${ }^{19}$

Finally, we give comments on the effects of free-carrier dynamics in CZTS on the solar-cell performance. In this study, we revealed the existence of long-lifetime free photocarriers and the slow energy relaxation of hot carriers in CZTS. The long lifetime of photocarriers, which is caused by the thermal excitation from the tail states, gives rise to a good charge collection observed in CZTS-based solar cells. $^{5,27}$ Therefore, we conclude that CZTS has a high potential for solar-cell materials. In addition, the slow picosecond relaxation of hot carriers might produce a positive effect on the power conversion efficiency of CZTS-based solar cells, because hot carriers with large excess energies result in efficient charge-separation and charge-transport. However, the deep defects existing in $\mathrm{CZTS}^{6-10}$ lead to the low open-circuit voltage and limit the power conversion efficiency of CZTS-based solar cells. ${ }^{5,27}$ In order to improve the performance of CZTS-based solar cells, more efforts must be made to reduce the deep defect states.

In conclusion, we experimentally studied the dynamics of free carriers in CZTS single crystals by a combination of TR and THz-TA measurements. The thermal excitation of photocarriers localized in the tail states results in the long lifetime of free carriers observed consistently in the TR and THz-TA decay dynamics. The slow picosecond energy relaxation of hot carriers in CZTS may be caused by electrostatic potential fluctuations near the band edge. Under highenergy photoexcitation, nonradiative recombinations near the surface region affect the optoelectronic properties of CZTS. Our findings provide a deep insight behind physics of the photovoltaic operation of CZTS-based solar cells.

This work was supported by JST-CREST and the Sumitomo Electric Industries Group CSR Foundation.

${ }^{1}$ D. B. Mitzi, O. Gunawan, T. K. Todorov, K. Wang, and S. Guha, Sol. Energy Mater. Sol. Cells 95, 1421 (2011).
${ }^{2}$ A. Polizzotti, I. L. Repins, R. Noufi, S.-H. Wei, and D. B. Mitzi, Energy Environ. Sci. 6, 3171 (2013).

${ }^{3}$ K. Ito and T. Nakazawa, Jpn. J. Appl. Phys., Part 1 27, 2094 (1988).

${ }^{4}$ L. Q. Phuong, M. Okano, Y. Yamada, A. Nagaoka, K. Yoshino, and Y. Kanemitsu, Appl. Phys. Lett. 103, 191902 (2013).

${ }^{5}$ B. Shin, O. Gunawan, Y. Zhu, N. A. Bojarczuk, S. J. Chey, and S. Guha, Prog. Photovoltaics Res. Appl. 21, 72 (2013).

${ }^{6}$ S. Chen, J. H. Yang, X. G. Gong, A. Walsh, and S. H. Wei, Phys. Rev. B 81, 245204 (2010).

${ }^{7}$ M. J. Romero, H. Du, G. Teeter, Y. Yan, and M. M. Al-Jassim, Phys. Rev. B 84, 165324 (2011).

${ }^{8}$ S. Levcenko, V. E. Tezlevan, E. Arushanov, S. Schorr, and T. Unold, Phys. Rev. B 86, 045206 (2012).

${ }^{9}$ T. Gokmen, O. Gunawan, T. K. Todorov, and D. B. Mitzi, Appl. Phys. Lett. 103, 103506 (2013).

${ }^{10}$ L. Q. Phuong, M. Okano, Y. Yamada, A. Nagaoka, K. Yoshino, and Y. Kanemitsu, Appl. Phys. Lett. 104, 081907 (2014).

${ }^{11}$ S.-H. Wei, S. B. Zhang, and A. Zunger, App. Phys. Lett. 72, 3199 (1998).

${ }^{12}$ Y. Yamada and Y. Kanemitsu, Appl. Phys. Lett. 101, 133907 (2012).

${ }^{13}$ Y. Yamada, T. Nakamura, M. Endo, A. Wakamiya, and Y. Kanemitsu, J. Am. Chem. Soc. 136, 11610 (2014).

${ }^{14}$ J. Shah, Ultrafast Spectroscopy of Semiconductors and Semiconductor Nanostructures (Springer, Berlin, 1999).

${ }^{15}$ R. Ulbricht, E. Hendry, J. Shan, T. F. Heinz, and M. Bonn, Rev. Mod. Phys. 83, 543 (2011).

${ }^{16}$ A. Nagaoka, K. Yoshino, H. Taniguchi, T. Taniyama, and H. Miyake, J. Cryst. Growth 341, 38 (2012).

${ }^{17}$ A. Nagaoka, H. Miyake, T. Taniyama, K. Kakimoto, and K. Yoshino, Appl. Phys. Lett. 103, 112107 (2013).

${ }^{18}$ G. Yamashita, E. Mastubara, M. Nagai, Y. Kanemitsu, and M. Ashida, "Intrinsic carrier multiplication efficiency in bulk Si crystals evaluated by optical-pump/terahertz-probe spectroscopy," Appl. Phys. Lett. (in press).

${ }^{19}$ M. Okano, Y. Takabayashi, T. Sakurai, K. Akimoto, H. Shibata, S. Niki, and Y. Kanemitsu, Phys. Rev. B 89, 195203 (2014).

${ }^{20}$ Y. Yamada, T. Nakamura, S. Yasui, H. Funakubo, and Y. Kanemitsu, Phys. Rev. B 89, 035133 (2014).

${ }^{21}$ Y. Yamada, T. Nakamura, M. Endo, A. Wakamiya, and Y. Kanemitsu, Appl. Phys. Express 7, 032302 (2014).

${ }^{22}$ C. Persson, J. Appl. Phys. 107, 053710 (2010).

${ }^{23}$ W. Z. Lin, R. W. Schoenlein, J. G. Fujimoto, and E. P. Ippen, IEEE J. Quantum Electron. 24, 267 (1988).

${ }^{24}$ A. Othonos, J. Appl. Phys. 83, 1789 (1998).

${ }^{25}$ A. P. Levanyuk and V. V. Osipov, Sov. Phys. Usp. 24, 187 (1981).

${ }^{26}$ I. Dirnstorfer, Mt. Wagner, D. M. Hofmann, M. D. Lampert, F. Karg, and B. K. Meyer, Phys. Status Solidi A 168, 163 (1998).

${ }^{27}$ W. Wang, M. T. Winkler, O. Gunawan, T. Gokmen, T. K. Todorov, Y. Zhu, and D. B. Mitzi, Adv. Energy Mater. 4, 1301465 (2014). 